\title{
Toxicidad crónica del Cadmio y sus efectos sobre actividad de TGO, TGP, creatinina y alteración histológica en riñón e hígado en Rattus norvegicus variedad SPRAGUE DAWLEY
}

Chronic toxicity of Cadmium and its effects on activity of TGO, TGP, creatinine and histological alteration in kidney and liver in Rattus norvegicus variety SPRAGUE DAWLEY.

Henry Díaz-Murillo

Universidad Nacional de San Agustín. Arequipa Perú.

\section{INFORMACIÓN}

\section{Historia del Artículo}

Recepción: 20/06/2019

Revisión: 17/07/2019

Aceptación: 22/07/2019

\section{Palabras Clave}

Toxicidad, Cadmio, hígado, creatinina, riñon, Rattus norvegicus.

\section{Key Words}

Cadmium, liver, creatinine, kidney, Rattus norvegicus.

\section{DOI}

https://doi.org/10.35286/veritas. v20i1.234

\begin{abstract}
RESUMEN
Se evaluó los efectos del cadmio sobre el peso corporal, la actividad de TGO, TGP, creatinina y los cambios histológicos en hígado y riñón de Rattus norvegicus variedad Sprague Dawley. La evaluación experimental se realizó en el Laboratorio de Fisiología Animal del Departamento Académico de Biología de la U.N.S.A.-Arequipa, durante los meses de Enero a Junio 2015.

Se utilizaron 24 Rattus norvegicus variedad Sprague Dawley asignados en cuatro grupos: Control (Agua destilada), Grupo 2 (5 mg/Kg de cloruro de cadmio), Grupo $3(10 \mathrm{mg} / \mathrm{Kg}$. de cloruro de cadmio) y Grupo 4 ( $20 \mathrm{mg} / \mathrm{Kg}$. de cloruro de cadmio) durante 4 semanas.

Se encontró diferencia significativa $(\mathrm{p}<0,05)$ en el peso corporal de las ratas en los diferentes grupos de evaluación. Al aplicar la prueba de postcomparación de Tukey se encontró que en el tratamiento con cloruro de cadmio en dosis de $20 \mathrm{mg} / \mathrm{kg} / \mathrm{día}$, los pesos corporales de las ratas intoxicadas disminuyeron significativamente. Se observó diferencia significativa $(p<0.01)$ en la actividad de TGO, TGP creatinina de las ratas en los diferentes grupos de evaluación. Al aplicar la prueba de postcomparación de Tukey, se encontró que en los tratamientos con cloruro de cadmio en dosis de 10 y $20 \mathrm{mg} / \mathrm{kg} /$ día los niveles de TGO, TGP y creatinina de las ratas intoxicadas se incrementaron significativamente. La exposición crónica de cloruro de cadmio provocó congestión leve con dilatación de los sinusoides hepáticos y alteración del arreglo arquitectural de los hepatocitos; congestión vascular y aumento de la celularidad glomerular de moderado a severo, degeneración hialina del epitelio tubular en el riñón de las ratas que se les administró cloruro de cadmio en dosis de $10,20 \mathrm{mg} / \mathrm{kg} /$ día durante 30 días.
\end{abstract}

\begin{abstract}
Cadmium effects on body weight, the activity of AST, ALT, and creatinine histological changes in the liver and testis Sprague Dawley Rattus norvegicus was evaluated. The experimental evaluation was performed at the Laboratory of Animal Physiology of the Academic Department of Biology of the UNSA Arequipa, during the months of January to June 2015. Control (Distilled water), Group 2 (5 mg / kg of cadmium chloride), Group 3 (. $10 \mathrm{mg} / \mathrm{kg}$ of cadmium chloride) and Group 4 (20: 24 Sprague Dawley Rattus norvegicus allocated in four treatment groups $\mathrm{mg}$ / Kg. of cadmium chloride) for 4 weeks. It significant difference $(\mathrm{p}<0.05)$ was found in body weight of rats in different groups of evaluation. When applying postcomparación Tukey test it was found that in the treatment with cadmium chloride at doses of $20 \mathrm{mg} / \mathrm{kg} /$ day, body weights of rats treated decreased significantly. It significant difference $(\mathrm{p}<0.01)$ was observed in the activity of SGOT, SGPT creatinine of rats in different groups of evaluation. When applying postcomparación Tukey test revealed that in treatments with cadmium chloride at doses of 10 and $20 \mathrm{mg} / \mathrm{kg} /$ day levels of GOT, GPT and creatinine intoxicated rats increased significantly. Chronic exposure to cadmium chloride caused congestion with mild hepatic sinusoidal dilation and alteration of architectural arrangement of hepatocytes; vascular congestion and increased glomerular cellularity of moderate to severe hyaline degeneration of tubular epithelium in rats that were administered cadmium chloride at doses of $10,20 \mathrm{mg} / \mathrm{kg} /$ day for 30 days.
\end{abstract}

\section{MATERIAL Y MÉTODOS}

\section{Lugar y fecha de Ejecución}

El presente trabajo de investigación se realizó en el Laboratorio del Área de Fisiología Animal del Departamento Académico de Biología - U.N.S.A. durante el periodo de Enero a Junio 2015

Correspondencia:

Henry Díaz-Murillo

hdiazmur@gmail.com

\section{Diseño experimental}

\begin{tabular}{cc}
\hline TRATAMIENTO & N $^{\circ}$ DE RATAS \\
\hline T1 Control & 6 \\
T2 Cloruro de cadmio $5 \mathrm{mg} / \mathrm{kg}$ & 6 \\
T3 Cloruro de cadmio $10 \mathrm{mg} / \mathrm{kg}$ & 6 \\
T4 Cloruro de cadmio $20 \mathrm{mg} / \mathrm{kg}$ & 6 \\
TOTAL & 24
\end{tabular}


Las dosis se administraron por treinta días de acuerdo a los tratamientos descritos.

\section{MATERIAL Y METODOLOGÍA}

Se utilizaron 24 Rattus norvegicus variedad Sprague Dawley, las que fueron alimentadas y tratadas por 30 días con dosis diarias de cloruro de cadmio en concentraciones de 5,10 y $20 \mathrm{mg} / \mathrm{kg}$ de peso corporal; para esto se utilizó una sonda orogástrica. Y cada 10 días fueron pesadas en una balanza analítica, colocándolas en cajas de inmovilización. Después se procedió a tomar muestras de sangre extraídas de la cola de las ratas de experimentación con la ayuda de una hoja de bisturí y un tubo capilar.

Se procedió a centrifugar los capilares, debidamente rotulados, para obtener suero y utilizarlo para el examen de fosfatasa ácida que se realizó cada 10 días.

Al finalizar el periodo de experimentación, las ratas fueron sacrificadas para obtener hígado y riñón para la evaluación histológica; los que fueron cubiertos con gasas y conservados en frascos con formol al $10 \%$, debidamente rotulados para cada tratamiento.

\section{DETERMINACIÓN DE TGO Y TGP}

(Método Reitman Frankel estándar del Reactivo VALTEK )

Las transaminasas se encuentran presentes en todos los tejidos, pero en altas concentraciones en el hígado, músculo, riñón y corazón. Su aumento se asocia a enfermedades que afectan dichos tejidos, tales como hepatitis, cirrosis, infarto al miocardio, etc.

Fundamento del Método

La determinación de la transaminasa glutámico oxalacética (GOT) se basa en la siguiente reacción:

TGO

Glutamato

L-Aspartato + A-Cetoglutarato Oxaloacetato +

La determinación de la transaminasa glutámico pirúvica (GPT) se basa en la siguiente reacción: TGP

Glutamato

L-Alanina + A-Cetoglutarato Piruvato +

El piruvato u oxalacetato formado reacciona con la 2,4-dinitrofenilhidrazina, generando en medio alcalino una hidrazona coloreada que absorbe a $505 \mathrm{~nm}$.

Reactivos

Conservados entre 2 y $8^{\circ} \mathrm{C}$, son estables hasta la fecha de caducidad indicada en la etiqueta.

\section{Sustrato TGP}

\begin{tabular}{|c|c|c|}
\hline $\begin{array}{l}\text { Buffer fosfato } \mathrm{pH} 7.4 \\
\text { L-Alanina }\end{array}$ & $100 \mathrm{mM}$ & $100 \mathrm{mM}$ \\
\hline 2-oxoglutarato & $2 \mathrm{mM}$ & \\
\hline Sustrato TGO & & \\
\hline Buffer fosfato $\mathrm{pH} 7.4$ & $100 \mathrm{mM}$ & \\
\hline L-aspartato & & $100 \mathrm{mM}$ \\
\hline $\begin{array}{l}\text { 2-oxoglutarato } \\
\text { Reactivo de Color }\end{array}$ & $2 \mathrm{mM}$ & \\
\hline $\begin{array}{l}\text { 2,4-dinitrofenilhidrazina } \\
\mathrm{HCl}\end{array}$ & $1 \mathrm{mM}$ & $1 \mathrm{mM}$ \\
\hline Standard & & \\
\hline Piruvato & & $2 \mathrm{mM}$ \\
\hline
\end{tabular}

\section{Muestra}

De preferencia utilizar suero libre de hemólisis. Descartar muestras con hemólisis visible y que se puedan obtener valores falsamente elevados. Las transaminasas son estables a lo menos 7 días entre 2 y $8^{\circ} \mathrm{C}$ y sobre un mes a $-20^{\circ} \mathrm{C}$.

\section{Equipo requerido}

Espectrofotómetro capaz de leer a $505 \mathrm{~nm}$ (rango 500 $550 \mathrm{~nm})$, baño termorregulado y pipetas.

\section{Técnica}

Curva de calibración

\begin{tabular}{cccccccc}
\hline TUBO & & 1 & 2 & 3 & 4 & 5 & 6 \\
Agua destilada & $(\mathrm{ml})$ & 0.2 & 0.2 & 0.2 & 0.2 & 0.2 & 0.2 \\
Standard & $(\mathrm{ml})$ & - & 0.1 & 0.2 & 0.3 & 0.4 & 0.5 \\
Sustrato TGO & $(\mathrm{ml})$ & 1.0 & 0.9 & 0.8 & 0.7 & 0.6 & 0.5 \\
Reactivo color & $(\mathrm{ml})$ & 1.0 & 1.0 & 1.0 & 1.0 & 1.0 & 1.0
\end{tabular}

Mezclar e incubar 20 minutos a temperatura ambiente (sobre $20^{\circ} \mathrm{C}$.)

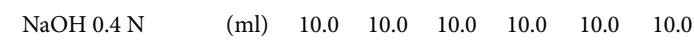
Mezclar, esperar 5 minutos y leer a $505 \mathrm{~nm}$, (rango $500-550 \mathrm{~nm}$.), contra \begin{tabular}{lllllllll}
\multicolumn{8}{l}{ blanco reactivo (tubo 1$)$} \\
TGO & (UI) & 0 & 22 & 55 & 95 & 150 & 215
\end{tabular} $\begin{array}{lllllll}\text { TGP (UI) } & 0 & 25 & 50 & 83 & 126\end{array}$ Graficar en papel milimetrado las unidades de transaminasas en la abcisas (eje X), y las absorbancias en las ordenadas (eje Y).

\section{Ensayo}

\begin{tabular}{|c|c|c|c|}
\hline & Blanco & TGO & TGP \\
\hline Sustrato TGO (ml) & 0.5 & 0.5 & - \\
\hline Sustrato TGP (ml) & - & - & 0.5 \\
\hline \multicolumn{4}{|c|}{ Incubar por 3 minutos a $37^{\circ} \mathrm{C}$} \\
\hline Agua destilada (ml) & 0.1 & - & - \\
\hline Muestra & - & 0.2 & 0.1 \\
\hline \multicolumn{4}{|c|}{ Incubar 30 minutos a $37^{\circ} \mathrm{C}$} \\
\hline Reactivo color (ml) & 0.5 & 0.5 & 0.5 \\
\hline \multicolumn{4}{|c|}{ Incubar 20 minutos a temperatura ambiente (sobre $20^{\circ} \mathrm{C}$ ) } \\
\hline $\mathrm{NaOH} 0.4 \mathrm{~N} \quad(\mathrm{ml})$ & 5.0 & 5.0 & 5.0 \\
\hline \multicolumn{4}{|c|}{$\begin{array}{c}\text { Mezclar por inversión y leer a } 505 \mathrm{~nm} .(500-550 \mathrm{~nm} \text {.) contra blanco reactivo } \\
\text { El color resultante es estable por } 30 \text { minutos. }\end{array}$} \\
\hline
\end{tabular}

\section{Resultados}

Determinar la actividad de las transaminasas por interpolación de las absorbancias obtenidas en la curva de calibración.

\section{Observaciones}

- Los volúmenes de reactivo y muestra pueden ser alterados proporcionalmente sin alterar los resultados.

- La temperatura y los tiempos de incubación son críticos, por tanto deben ser respetados fielmente.

- Es recomendable realizar un blanco muestra (sin incubar y agregando el suero después del reactivo color), en aquellas muestras muy hemolíticas, ictéricas o en las que se sospeche de cetosis (Karmen, 1955; Reitman y Frankel, 1957; Henry, 1964). 
Valor normal para ratas (según Altman y Raymund, 1969)

TGO: Valor referencial $(5-80 \mathrm{U} / \mathrm{l})$

TGP: Valor referencial ( 5 - $50 \mathrm{U} / \mathrm{l})$

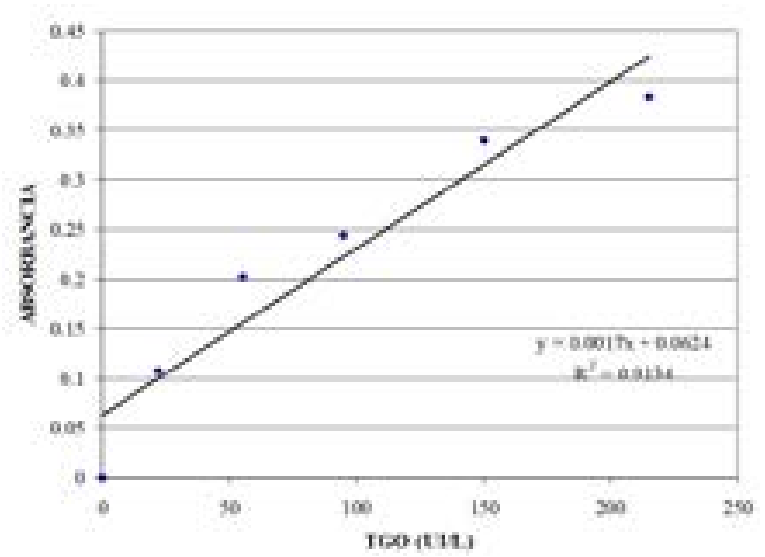

CURVA DE CALIBRACIÓN DE TGO (UI/L)

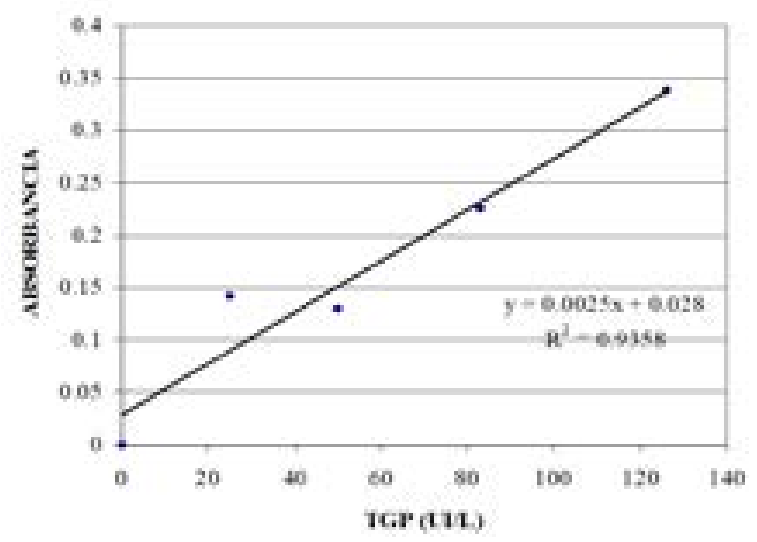

CURVA DE CALIBRACIÓN DE TGO (UI/L)

\section{Determinación de creatinina}

Las muestras de sangre se obtuvieron por el método de Archer extraídas de la cola de los animales de experimentación con la ayuda de una hoja de bisturí y un tubo capilar.

\section{TECNICA DE PUNTO FINAL Método de Owen}

La creatinina reacciona con el picrato alcalino en medio tamponado, previa desproteinización con ácido pícrico, obteniéndose un cromógeno que se mide a $510 \mathrm{~nm}$

\section{MUESTRA: Suero}

\section{Condiciones de reacción}

- Longitud de onda: $510 \mathrm{~nm}$ en espectrofotómetro

- Temperatura de reacción: $37^{\mathrm{a}} \mathrm{C}$.

- Tiempo de reacción: 15 minutos.

- Volumen de muestra: $0.1 \mathrm{ml}$

- Volumen de Reactivo: $1 \mathrm{ml}$

- Volumen final de reacción: $1.15 \mathrm{ml}$.

\section{Procedimiento}

En tres tubos marcados B (blanco), S (Standard) y D (desconocido) colocar:

\begin{tabular}{cccc}
\hline & B & S & D \\
\hline Agua destilada & $100 \mathrm{ul}$ & --- & --- \\
Standard & --- & $100 \mathrm{ul}$ & --- \\
Muestra & --- & --- & $100 \mathrm{ul}$ \\
Reactivo de trabajo & $1 \mathrm{ml}$ & $1 \mathrm{ml}$ & $1 \mathrm{ml}$ \\
\hline
\end{tabular}

Mezclar. Incubar 10 minutos en baño Maria a $37^{\mathrm{a}} \mathrm{C}$. Dentro de los $15 \mathrm{minu}$ tos de retirado del baño, leer el Standard y el desconocido (D1) en espectrofotómetro a $510 \mathrm{~nm}$ llevando a 0.000 D.O. con el blanco. Luego agregar:

\begin{tabular}{ccc}
\hline Stopper & $50 \mathrm{ul}$ & $50 \mathrm{ul}$ \\
\hline $\begin{array}{c}\text { Mezclar. Dejar los tubos } 5 \text { minutos a temperatura ambiente y volver a leer el } \\
\text { Desconocido (D2), llevando a }\end{array}$ \\
\hline
\end{tabular}

\section{Estabilidad de la mezcla de reacción final}

El color de la reacción es estable durante 6 horas por lo que la absorbancia debe ser leída dentro de ese lapso

\section{Calculo de los resultados}

Creatinina en suero $(\mathrm{mg} / \mathrm{l})=(\mathrm{D} 2-\mathrm{D} 1) \times \mathrm{f}$

$$
\mathrm{f}=20 \mathrm{mg}
$$

$\mathrm{S}$

Valor normal para ratas (según Altman y Raymund, 1969) Creatinina: Valor referencial $(0.4-0.95 \mathrm{mg} / \mathrm{dl})$

\section{Evaluación histológica \\ Obtención de la Muestra}

Después de sacrificado el animal, inmediatamente se procedió a extraer de ellos hígado y riñón para proceder al estudio histopatológico.

\section{RESULTADOS}

Se evaluó el efecto tóxico del cloruro de cadmio en Rattus norvegicus variedad Sprague Dawley. Los resultados obtenidos se muestran en Tablas y Figuras 
Tabla 1: PESO PROMEDIO (g) DE Rattus norvegicus VARIEDAD SPRAGUE DAWLEY TRATADAS CON CLORURO DE CADMIO DURANTE 30 DIAS

\begin{tabular}{ccccc}
\hline \multirow{2}{*}{ TRATAMIENTOS } & \multicolumn{4}{c}{ PESO CORPORAL $(\mathrm{g})$} \\
\cline { 2 - 5 } CONTROL & BASAL & 10 & 20 & 30 \\
Cl2Cd 5mg/kg & $261.4^{\mathrm{a}}$ & $276.2^{\mathrm{b}}$ & $307.3^{\mathrm{c}}$ & $315.2^{\mathrm{d}}$ \\
Cl2Cd $10 \mathrm{mg} / \mathrm{kg}$ & $265.3^{\mathrm{a}}$ & $264.3^{\mathrm{a}}$ & $264.0^{\mathrm{b}}$ & $256.4^{\mathrm{c}}$ \\
Cl2Cd 20mg/kg & $264.1^{\mathrm{a}}$ & $263.6^{\mathrm{a}}$ & $268.6^{\mathrm{b}}$ & $231.5^{\mathrm{b}}$ \\
\hline F (ANOVA) & 0,41 & 6.21 & $254.2^{\mathrm{a}}$ & $219.5^{\mathrm{a}}$ \\
P & $>0.05$ & $<0.05$ & $<0.01$ & $<0.01$ \\
SIGNIFICANCIA & N.S & $\mathrm{S}$ & A.S & A.S. \\
\hline
\end{tabular}

${ }^{\text {abc TUKEY }}(\mathrm{P}<0.05)$

En la Tabla y Gráfico 1 se muestra que existe diferencia significativa $(p<0.05)$ en el peso corporal de las ratas en los diferentes grupos de evaluación. Al aplicar la prueba de postcomparación de Tukey se encontró que el tratamiento con cloruro de cadmio en dosis de 5, 10 y $20 \mathrm{mg} / \mathrm{kg} / \mathrm{día}$ los pesos corporales de las ratas intoxicadas disminuyeron significativamente $(\mathrm{p}<0.05)$.

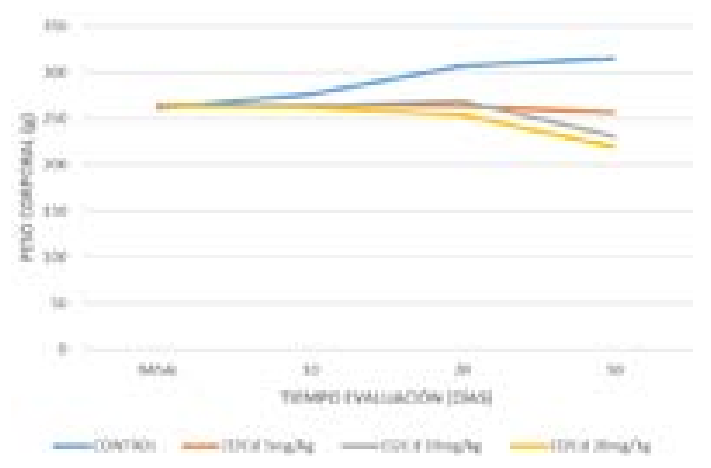

Graf. 1: PESO PROMEDIO (g) DE Rattus norvegicus VARIEDAD SPRAGUE DAWLEY TRATADAS CON CLORURO DE CADMIO DURANTE 30 DIAS

Tabla 2: TGO (UI/L) DE Rattus norvegicus VARIEDAD SPRAGUE DAWLEY TRATADAS CON CLORURO DE CADMIO DURANTE 30 DIAS

\begin{tabular}{ccccc}
\hline \multirow{2}{*}{ TRATAMIENTOS } & \multicolumn{4}{c}{ TGO (UI/L) } \\
\cline { 2 - 5 } CONTROL & BASAL & 10 & 20 & 30 \\
Cl2Cd 5mg $/ \mathrm{kg}$ & $65.36 \mathrm{a}$ & $81.02 \mathrm{ab}$ & $68.66^{\mathrm{a}}$ & $79.83^{\mathrm{a}}$ \\
$\mathrm{Cl} 2 \mathrm{Cd} 10 \mathrm{mg} / \mathrm{kg}$ & $62.16 \mathrm{a}$ & $83.42 \mathrm{ab}$ & $77.81 \mathrm{ab}$ & $84.25 \mathrm{ab}$ \\
$\mathrm{Cl} 2 \mathrm{Cd} 20 \mathrm{mg} / \mathrm{kg}$ & $62.84 \mathrm{a}$ & $83.03 \mathrm{~b}$ & $89.96 \mathrm{~b}$ & $89.10 \mathrm{~b}$ \\
\hline F $($ ANOVA) & 2.01 & 3.37 & 7.02 & $100.60 \mathrm{c}$ \\
P & $>0.05$ & $<0.05$ & $<0.01$ & $<0.01$ \\
SIGNIFICANCIA & NS & $\mathrm{S}$ & AS & AS \\
\hline
\end{tabular}

${ }^{a b c}$ TUKEY $(\mathrm{P}<0.05)$ TGO: Valor referencial en ratas $(5-80$ $\mathrm{U} / 1)$
En la Tabla y Gráfico 2 se muestra que existe diferencia significativa $(\mathrm{p}<0.01)$ en los niveles de TGO de las ratas en los diferentes grupos de evaluación. Al aplicar la prueba de postcomparación de Tukey se encontró que en los tratamiento con cloruro de cadmio en dosis de 5,10 y $20 \mathrm{mg} / \mathrm{kg} / \mathrm{día}$ la actividad de TGO de las ratas intoxicadas se incrementó significativamente $(\mathrm{p}<0.01)$.



Graf. 2: TGO (UI/L) DE Rattus norvegicus VARIEDAD SPRAGUE DAWLEY TRATADAS CON CLORURO DE CADMIO DURANTE 30 DIAS

Tabla 3: TGP (UI/L) DE Rattus norvegicus VARIEDAD SPRAGUE DAWLEY TRATADAS CON CLORURO DE CADMIO DURANTE 30 DIAS

\begin{tabular}{ccccc}
\hline \multirow{2}{*}{ TRATAMIENTOS } & \multicolumn{4}{c}{ TGP (UI/L) } \\
\cline { 2 - 5 } CONTROL & BASAL & 10 & 20 & 30 \\
Cl2Cd 5mg/kg & $37.90^{\mathrm{a}}$ & $41.66^{\mathrm{a}}$ & $44.83 \mathrm{a}$ & $44.15^{\mathrm{a}}$ \\
Cl2Cd 10mg/kg & $39.75^{\mathrm{a}}$ & $46.79 \mathrm{~b}$ & $46.59 \mathrm{a}$ & $49.71^{\mathrm{a}}$ \\
Cl2Cd 20mg $/ \mathrm{kg}$ & $37.79^{\mathrm{a}}$ & $45.98 \mathrm{ab}$ & $47.80 \mathrm{a}$ & $85.04 \mathrm{~b}$ \\
\hline F (ANOVA) & 1.08 & 4.09 & 29.61 & 174.54 \\
P & $>0.05$ & $<0.05$ & $<0.01$ & $<0.01$ \\
SIGNIFICANCIA & NS & $\mathrm{S}$ & AS & AS \\
\hline
\end{tabular}

abc TUKEY $(\mathrm{P}<0.05)$ TGP: Valor referencial en ratas ( 5 - 50 $\mathrm{U} / 1)$

En la Tabla y Gráfico 2 se muestra que existe diferencia significativa $(p<0.01)$ en los niveles de TGP de las ratas en los diferentes grupos de evaluación. Al aplicar la prueba de postcomparación de Tukey se encontró que en los tratamiento con cloruro de cadmio en dosis de 10 y $20 \mathrm{mg} / \mathrm{kg} /$ día la actividad de TGP de las ratas intoxicadas se incrementó significativamente $(\mathrm{p}<0.01)$.

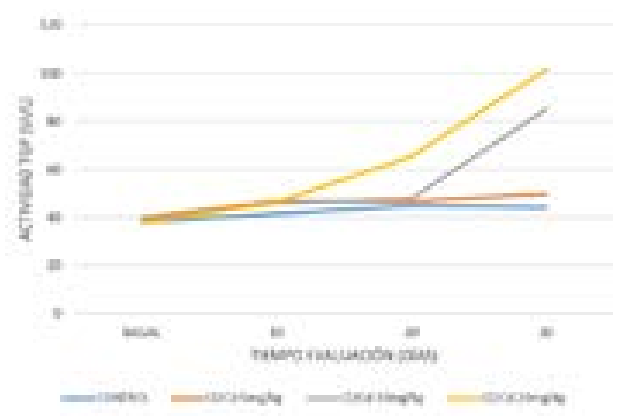

Graf. 3: TGP (UI/L) DE Rattus norvegicus VARIEDAD SPRAGUE DAWLEY TRATADAS CON CLORURO DE CADMIO DURANTE 30 DIAS 
Tabla 4: CREATININA (mg/dl) DE Rattus norvegicus VARIEDAD SPRAGUE DAWLEY TRATADAS CON CLORURO DE CADMIO DURANTE 30 DIAS

\begin{tabular}{ccccc}
\hline \multirow{2}{*}{ TRATAMIENTOS } & \multicolumn{4}{c}{ CREATININA (mg/dl)) } \\
\cline { 2 - 5 } CONTROL & BASAL & 10 & 20 & 30 \\
Cl2Cd 5mg/kg & $0,375^{\mathrm{a}}$ & $0,378^{\mathrm{a}}$ & $0,361^{\mathrm{a}}$ & $0,336^{\mathrm{a}}$ \\
$\mathrm{Cl} 2 \mathrm{Cd} 10 \mathrm{mg} / \mathrm{kg}$ & $0,356^{\mathrm{a}}$ & $0,385^{\mathrm{a}}$ & $0,456 \mathrm{~b}$ & $0,596 \mathrm{~b}$ \\
$\mathrm{Cl} 2 \mathrm{Cd} 20 \mathrm{mg} / \mathrm{kg}$ & $0,348^{\mathrm{a}}$ & $0,496 \mathrm{~b}$ & $0,621 \mathrm{c}$ & $0,884 \mathrm{c}$ \\
\hline F (ANOVA) & 0.97 & $0,778 \mathrm{c}$ & $0,872 \mathrm{~d}$ & $1,172 \mathrm{~d}$ \\
P & $>0.05$ & 408.6 & 457.2 & 795.8 \\
SIGNIFICANCIA & NS & $\mathrm{S}$ & AS & AS \\
\hline
\end{tabular}

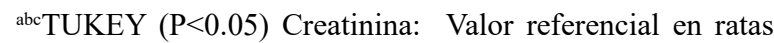
$(0.4-0.95 \mathrm{mg} / \mathrm{dl})$

En la Tabla y Gráfico 4 se muestra que existe diferencia significativa $(\mathrm{p}<0.01)$ en los niveles de creatinina de las ratas en los diferentes grupos de evaluación. Al aplicar la prueba de postcomparación de Tukey se encontró que en los tratamiento con cloruro de cadmio en dosis de 10 y $20 \mathrm{mg} /$ $\mathrm{kg}$ /día los niveles de creatinina de las ratas intoxicadas se incrementó significativamente $(\mathrm{p}<0.01)$.

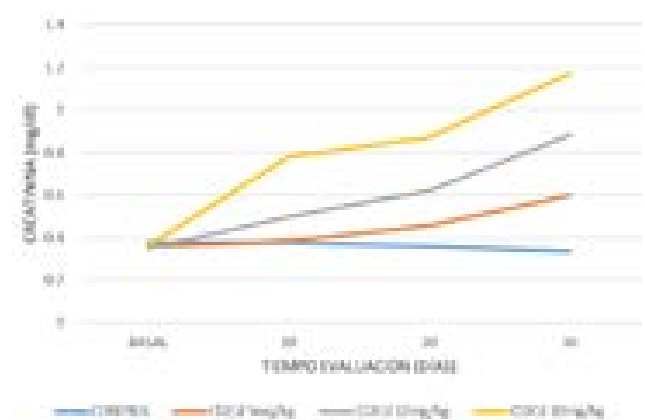

Graf. 4: CREATININA (mg/dl) DE Rattus norvegicus VARIEDAD SPRAGUE DAWLEY TRATADAS CON CLORURO DE CADMIO DURANTE 30 DIAS

\section{Evaluación histológica}

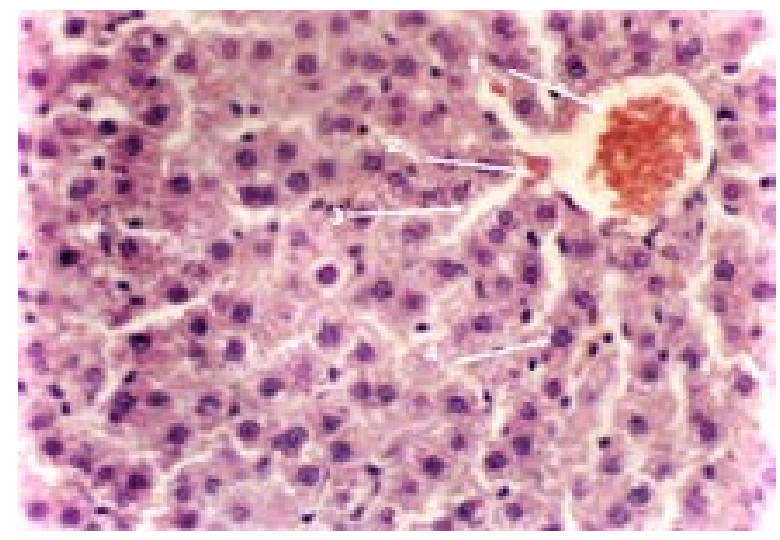

Fig 1: ESTRUCTURA DE HÍGADO DEL GRUPO CONTROL DE Rattus norvegicus VARIEDAD SPRAGUE DAWLEY ( HEMATOXILINA/ EOSINA $400 \mathrm{X}$ )
En esta microfotografía observamos la citoarquitectura normal de hígado con características normales en ratas del grupo control (grado 0).
1: Vena portal
2. Arteriola hepática
3: Sinusoides
4: Hepatocitos

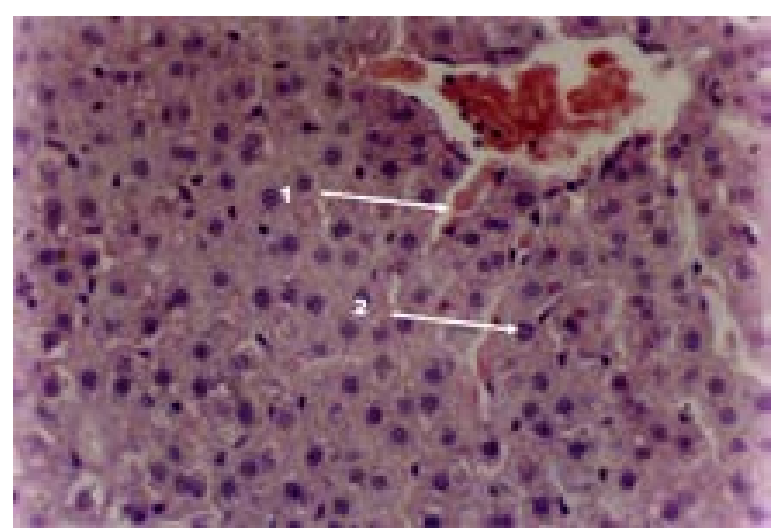

Fig 2: ESTRUCTURA DE HIGADO CON TRATAMIENTO DE CLORURO DE CADMIO EN DOSIS DE $5 \mathrm{mg} / \mathrm{kg} /$ día DE Rattus norvegicus VARIEDAD SPRAGUE DAWLEY POR 30 DIAS (HEMATOXILINA/ EOSINA $400 \mathrm{X}$ )

En esta microfotografía observamos el tejido hepático con alteraciones que van desde congestión leve con dilatación de los sinusoides hepáticos y alteración del arreglo arquitectural de los hepatocitos en las ratas expuestas a cloruro de cadmio a dosis de $5 \mathrm{mg} / \mathrm{kg} / \mathrm{día}$

1: Congestión y dilatación de los sinusoides hepáticos

2: Alteración de la citoarquitectura de los hepatocitos



Fig 3: ESTRUCTURA DE HÍGADO CON TRATAMIENTO DE CLORURO DE CADMIO EN DOSIS DE $10 \mathrm{mg} / \mathrm{kg} / \mathrm{d}$ dí EN Rattus norvegicus VARIEDAD SPRAGUE DAWLEY POR 30 DIAS (HEMATOXILINA/ EOSINA $400 \mathrm{X}$ )

En esta microfotografía observamos el tejido hepático con alteraciones que van desde congestión leve con dilatación de los sinusoides hepáticos y alteración del arreglo arquitectural de los hepatocitos en las ratas expuestas a cloruro de cadmio a dosis de $5 \mathrm{mg} / \mathrm{kg} /$ día

1: Congestión y dilatación de los sinusoides hepáticos

2: Alteración de la citoarquitectura de los hepatocitos 


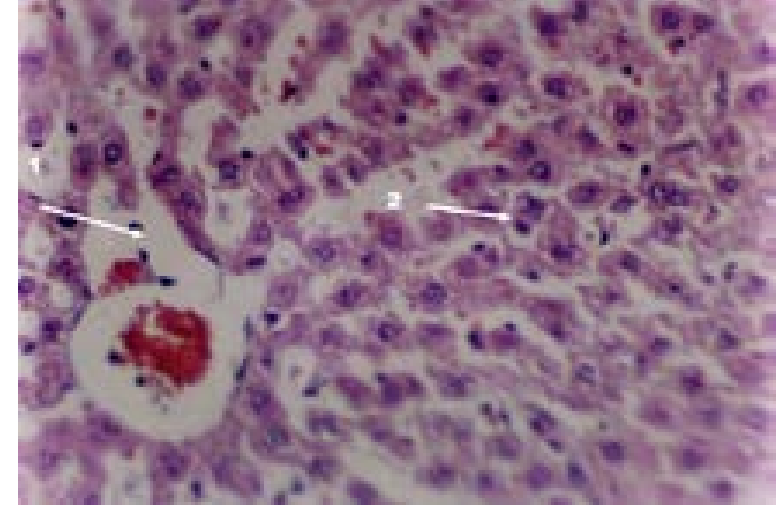

Fig 4: ESTRUCTURA DE HÍGADO CON TRATAMIENTO DE CLORURO DE CADMIO EN DOSIS DE $20 \mathrm{mg} / \mathrm{kg} / \mathrm{día}$ DE Rattus norvegicus VARIEDAD SPRAGUE DAWLEY POR 30 DIAS (HEMATOXILINA/EOSINA 400X)

En esta microfotografía observamos el tejido hepático con alteraciones que van desde congestión leve con dilatación de los sinusoides hepáticos y alteración del arreglo arquitectural de los hepatocitos en las ratas expuestas a cloruro de cadmio a dosis de $20 \mathrm{mg} / \mathrm{kg} /$ día

1: Congestión y dilatación de los sinusoides hepáticos

2: Alteración de la citoarquitectura de los hepatocitos

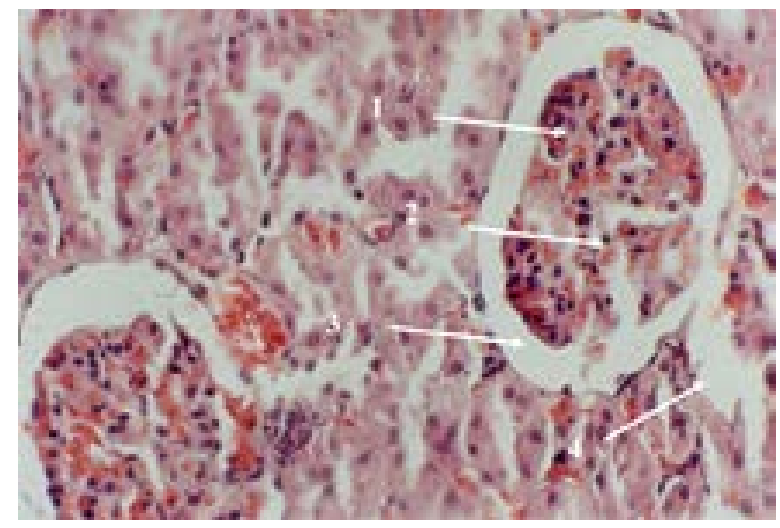

Fig 5: ESTRUCTURA DE RIÑÓN DEL GRUPO CONTROL DE Rattus norvegicus VARIEDAD SPRAGUE DAWLEY (HEMATOXILINA/EOSINA 400X)

En esta microfotografía observamos la citoarquitectura normal de riñón de ratas del grupo control. Se puede observar:

1: Capilares glomerulares

2: Glomérulo de Malpighi

3: Capsula de Bowman

4. Túbulos contorneado proximal

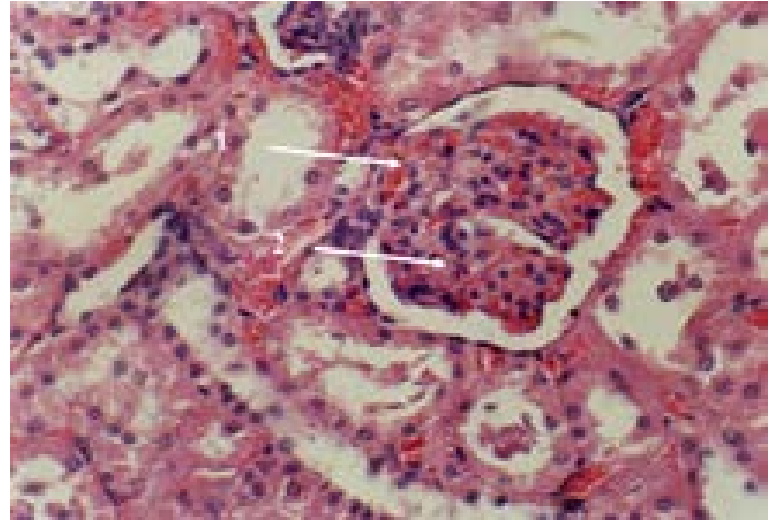

Fig 6: ESTRUCTURA DE RIÑón DE Rattus norvegicus VARIEDAD SPRAGUE DAWLEY CON TRATAMIENTO DE CLORURO DE CADMIO EN DOSIS DE 5 $\mathrm{mg} / \mathrm{kg} /$ día POR 30 DIAS (HEMATOXILINA/EOSINA 400X)

En la microfotografía observamos a nivel glomerular, congestión moderada, aumento moderado de la celularidad en ratas tratadas con cloruro de cadmio ( $5 \mathrm{mg} / \mathrm{kg} /$ día $)$.

1: Capilar congestionado

2: Aumento de celularidad

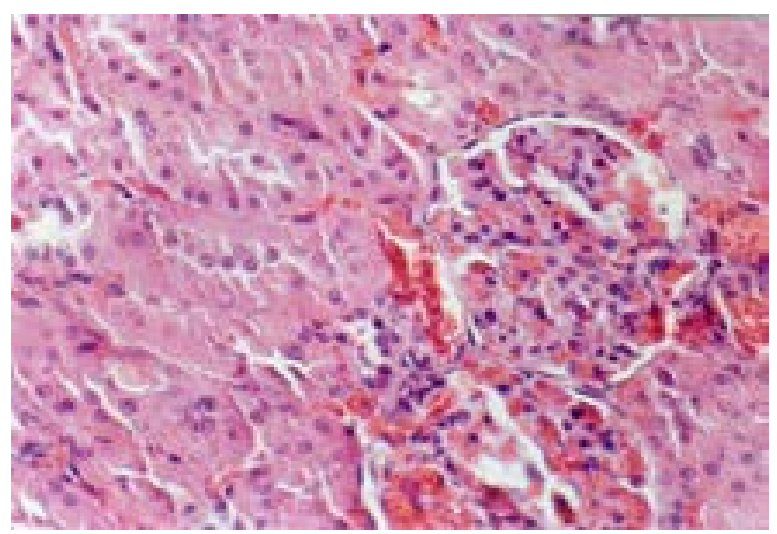

Fig 7: ESTRUCTURA DE RIÑóN DE Rattus norvegicus VARIEDAD SPRAGUE DAWLEY CON TRATAMIENTO DE CLORURO DE CADMIO EN DOSIS DE 10 mg/kg/día POR 30 DIAS (HEMATOXILINA/EOSINA 400X)

En esta microfotografía observamos a nivel glomerular, congestión severa, aumento moderado de la celularidad y alteración del capilar glomerular; a nivel del túbulo se observa, congestión severa y degeneración hialina en forma parcial en las ratas con dosis de cloruro de cadmio $10 \mathrm{mg} /$ $\mathrm{kg} / \mathrm{día}$

1: Congestión severa en el glomérulo

2: Alteración del capilar glomerular

3: Degeneración hialina del túbulo 


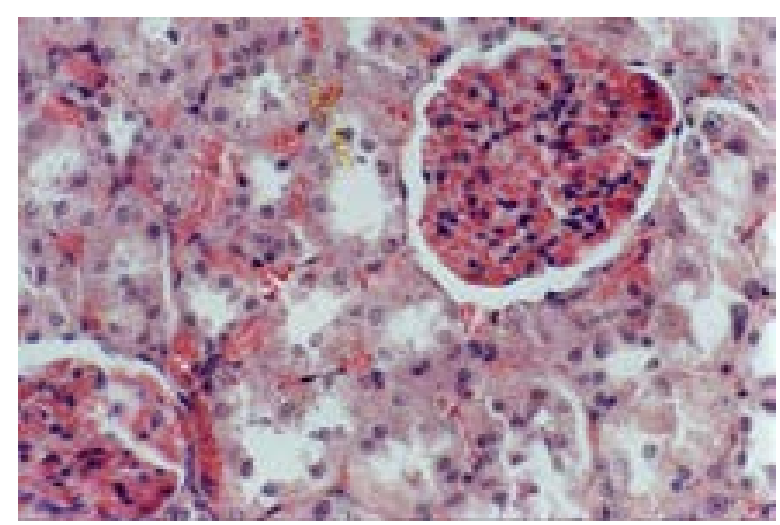

Fig 8: ESTRUCTURA DE RIÑÓN DE Rattus norvegicus VARIEDAD SPRAGUE DAWLEY CON TRATAMIENTO DE CLORURO DE CADMIO EN DOSIS DE 20 $\mathrm{mg} / \mathrm{kg} /$ día POR 30 DIAS (HEMATOXILINA/EOSINA 400X)

En esta microfotografía de riñón observamos congestión vascular y aumento de la celularidad glomerular de moderado a severo, degeneración hialina del epitelio tubular en ratas tratadas con dosis de $20 \mathrm{mg} / \mathrm{kg} /$ día de cloruro de cadmio.

1. Congestión vascular

2. Aumento de la celularidad

3 Degeneración hialina

\section{DISCUSIÓN}

Los mecanismos moleculares de la toxicidad de cadmio no son completamente conocidos hasta el momento; resultados obtenidos de animales en experimentación han mostrado que el cadmio puede interactuar con transportadores de membrana involucrados en la captura de metales esenciales tales como hierro y zinc en el tracto gastrointestinal, logrando desplazar a estos metales ingresando al citoplasma celular; esto ocurre debido a que el cadmio es capaz de mimetizar a estos cationes divalentes en el sitio de unión de uno o más acarreadores de proteínas y/o canales que transportan estos metales; ocasionando que el $\mathrm{Cd} 2+$ pueda formar un complejo coordinado covalente con ciertas biomoléculas que contienen grupos sulfhidrilo tales como el glutatión o cisteina; asimismo una de las principales entradas del cadmio a la célula está dada por los canales de calcio, tomando en cuenta que $\mathrm{Cd} 2+\mathrm{y}$ el calcio tienen un radio iónico similar, el metal puede introducirse libremente por esta vía a las células. Una de las proteínas importantes en una intoxicación por cadmio es la metalotioneina; esta proteína de bajo peso molecular y rica en cisteínas se encuentra presente en todos los tejidos, principalmente en hígado y riñones (donde se acumula más cadmio en el organismo), funciona principalmente atrapando iones de cadmio; asimismo, se encuentra en los astrocitos en el cerebro donde su función además de atrapar al cadmio es una enzima antioxidante. La alteración en la homeostasis del calcio intracelular lleva a la célula a una liberación del calcio mitocondrial y retículo endoplásmico; produciendo alteraciones en el metabolismo, interfiriendo con vías de señalización dependientes de calcio, con señales de transducción entre las células, daño a las membranas, bloqueo de canales dependientes de voltaje, regulación génica y bloqueo de la liberación de neurotransmisores. La liberación de neurotransmisores tales como serotonina y norepinefrina se ve alterado en animales en desarrollo que han sido expuestos al cadmio; asimismo, se sabe que el cadmio puede modificar el contenido de taurina y GABA en el hipotálamo, estriado y corteza prefrontal de ratas en desarrollo (Docampo y col. 2003).

Al evaluar la toxicidad del cloruro de cadmio en ratas de experimentación con 30 días de tratamiento, se encontró diferencia significativa $(\mathrm{p}<0.05)$ en el peso corporal de las ratas en los diferentes grupos de evaluación. Al aplicar la prueba de postcomparación de Tukey se encontró que en el tratamiento con cloruro de cadmio en dosis de 5, 10 y $20 \mathrm{mg} / \mathrm{kg} /$ día los pesos corporales de las ratas intoxicadas disminuyeron significativamente $(\mathrm{p}<0.05)$. Estos resultados coinciden con lo reportado por García, 2008 y Lara y col. 2003; quienes encontraron disminución en el peso corporal en ratas Wistar intoxicadas crónicamente con cloruro de cadmio. La disminución de peso observada provocaría hiperclorhídria, edema, hiperemia y erosión de la mucosa gastrointestinal lo que desencadenaría en un síndrome de mala absorción (Rubin y Farber, 2009).

Se observó diferencia significativa $(\mathrm{p}<0.01)$ en la actividad de la TGO y TGP de las ratas en los diferentes grupos de evaluación. Al aplicar la prueba de postcomparación de Tukey se encontró que en los tratamiento con cloruro de cadmio en dosis de 5, 10 y $20 \mathrm{mg} / \mathrm{kg} /$ día la TGO, TGP de las ratas intoxicadas incrementaron significativamente $(p<0.01)$. Este incremento de la actividad TGO y TGP nos indicaría una disfunción cardíaca y hepática, lo que se corroboraría con lo reportado por Obianime realizado en el 2009 quien trabajó en ratas Wistar encontrando aumento en los niveles de TGO y TGP en dosis crónicas de cloruro de cadmio. El mecanismo probable para la disfunción hepática y cardiaca podría deberse a que el cadmio provocaría un estrés oxidativo;- es decir, existe tanto un aumento en la velocidad de generación de especies reactivas del oxígeno como una disminución de los sistemas de defensa, lo que resulta en una mayor concentración, en estado estacionario, de radicales superóxidos. Es en esta situación de estrés oxidativo en la que se manifiestan las lesiones que producen los radicales libres. Estos reaccionan químicamente con lípidos, proteínas, carbohidratos y ADN al interior de las células hepáticas, y con componentes de la matriz extracelular, por lo que pueden desencadenar un daño irreversible que, si es muy extenso, puede llevar a la necrosis celular Rubin y Farber, (2009); Chen y col (2015), El Sokkary y col (2010), Madejczyk y $\operatorname{col}(2015)$.

Se observó diferencia significativa $(\mathrm{p}<0.01)$ en los niveles de creatinina de las ratas en los diferentes grupos de evaluación. Al aplicar la prueba de postcomparación de Tukey se encontró que en los tratamiento con cloruro de cadmio en dosis de 5,10 y $20 \mathrm{mg} / \mathrm{kg} /$ día los niveles de creatinina de las ratas intoxicadas incrementaron significativamente $(\mathrm{p}<0.01)$. Este incremento de los niveles de creatinina nos indicaría una insuficiencia renal, lo que se corroboraría con lo reportado Borky y col (2010), Obianine y col (2009), Lee y col (2014), Wallain y col (2014), Yan y col (2015).

En las figuras 1, 2, 3 y 4 se observó alteraciones que van desde congestión leve con dilatación de los sinusoides hepáticos y alteración del arreglo arquitectural de los hepatocitos en las ratas que se les administró cloruro de cadmio en dosis de 5,10 y $20 \mathrm{mg} / \mathrm{kg} /$ día durante 30 días. Estos resultados se corroboran con lo reportado por Chen y col. (2015). Dichos autores reportan daños irreversibles a nivel hepático con dosis crónicas de cloruro de cadmio en 
ratas Wistar. Estos resultados se deberían a que el cadmio potencia la apoptosis por estrés oxidativo en hígado Bork y col. (2010).

En las figuras 5, 6, 7 y 8 se observó alteraciones en riñón que van desde congestión vascular y aumento de la celularidad glomerular de moderado a severo, degeneración hialina del epitelio tubular en las ratas que se les administró cloruro de cadmio en dosis de 5,10 y $20 \mathrm{mg} / \mathrm{kg} /$ día durante 30 días. Estos resultados coinciden con lo reportado por Lee y col (2014), reportan que el cadmio libre se acumula en la mitocondria inhibiendo la cadena respiratoria en el complejo III; esto resulta en disfunción mitocondrial y formación de radicales libres, lo cual activa las enzimas caspasas y el desarrollo de apoptosis. También se une a los grupos sulfhidrilo de las proteínas afectando su estructura y función. Además señalan que el cadmio libre interfiere con las actividades enzimáticas del complejo calcio/calmodulina, inhibe la acción de la Na-K-ATPasa y estimula la actividad de las MAPcinasas. En las uniones paracelulares, afecta la distribución de las proteínas de la unión paracelular y disminuye la resistencia transepitelial Lee y col (2014), Wallain y col (2014), Yan y col (2015).

Las observaciones halladas en otros trabajos de investigación citan: insuficiencia renal, porque el cadmio se reabsorbe en los túbulos renales proximales, lo que desencadena una intensa proteinuria tubular, que puede resultar en una excreción de proteínas totales, 10 veces superior a lo normal para proteínas totales y hasta 1000 veces para las de bajo peso molecular como la beta 2 microglobulina (Docampo 2003).

Diversos estudios proponen a la apoptosis como el proceso dominante que participa en el efecto citotóxico inducido por el cadmio (Hamada y col. 1997; Lasfer y col., 2008), en cambio, otros autores le dan mayor preponderancia a la necrosis (Sancho y col., 2006; Brama y col., 2007).

La generación de estrés oxidativo es uno de los mecanismos más aceptados que explican el efecto citotóxico del cadmio (López y col., 2006; Ognjanović y col., 2008; Cuypers y col., 2010) y ambos tipos de muerte celular, apoptosis y necrosis, pueden ser desencadenados por este mecanismo (Fleury y col., 2002).

\section{REFERENCIAS BIBLIOGRÁFICAS}

1. ACHARYA UR, MISHRA M, PATRO J, PANDA MK. Effect of vitamins $\mathrm{C}$ and $\mathrm{E}$ on spermatogenesis in mice exposed to cadmium. Reprod Toxicol. 2008 Jan;25(1):84-8.

2. ASAGBA SO. Comparative effect of water and foodchain mediated cadmium exposure in rats. Biometals. 2010 Feb 27.

3. BARREGARD L, BERGSTRÖM G, FAGERBERG B. Cadmium, type 2 diabetes, and kidney damage in a cohort of middle-aged women. Environ Res. 2014 Nov;135:311-6.

4. BENEDETTI J L, SAMUEL O, DEWAILLY E, GINGRAS S AND LEFEBVRE M A, 1999. Levels of cadmium in kidney and liver tissues among a canadian population (Province of Quebec). J Toxicol Environ Health A 56(3):145-163.

5. BENITEZ MA, MENDEZ-ARMENTA M, MONTES S, REMBAO D, SANIN LH, RIOS C. Mother-fetus transference of lead and cadmium in rats: involvement of metallothionein. Histol Histopathol. 2009 Dec;24(12):1523-30.

6. BEVERIDGE R, PINTOS J, PARENT M E, ASSELIN J AND SIEMIATYCKI J, 2010. Lung cancer risk associated with occupational exposure to nickel, chromium VI, and cadmium in two populationbased case-control studies in Montreal. Am J Ind Med 53(5):476-85.

7. BHATTACHARYYA M H, Wilson A K, Rajan S S and Jonah M, 2000. Biochemical pathways in cadmium toxicity. In: Molecular Biology and Toxicology of Metals. Zalup R K, Koropatnick J, (eds), Taylor and Francis, London: 1-74.

8. BISHAK YK, PAYAHOO L, OSATDRAHIMI A, NOURAZARIAN A. Mechanisms of cadmium carcinogenicity in the gastrointestinal tract. Asian Pac J Cancer Prev. 2015;16(1):9-21.

9. BISWAS NM, SEN GUPTA R, CHATTOPADHYAY A, CHOUDHURY GR, SARKAR M. Effect of atenolol on cadmium-induced testicular toxicity in male rats. Reprod Toxicol. 2001 Nov-Dec;15(6):699-704.

10. BORK U, LEE WK, KUCHLER A, DITTMAR T, THÉVENOD F. Cadmium-induced DNA damage triggers $\mathrm{G}(2) / \mathrm{M}$ arrest via chk1/2 and cdc2 in p53deficient kidney proximal tubule cells. Am J Physiol Renal Physiol. 2010 Feb;298(2):F255-65.

11. BRAMA M, GNESSI L, BASCIANI S, CERULLI N, POLITI L, SPERA G, MARIANI S, CHERUBINI S, D'ABUSCO AS, SCANDURRA R AND MIGLIACCIO S, 2007. Cadmium induces mitogenic signaling in breast cancer cell by an ERalpha dependent mechanism. Mol Cell Endocrinol 264:102-108.

12. CALLEGARO MG, MILBRADT BG, DIETTRICH T, ALVES E, DUARTE FA, FLORES EM, DRESSLER VL, SILVA LP, EMANUELLI T. Influence of cereal bran supplement on cadmium effects in growing rats. Hum Exp Toxicol. 2009 Dec 17.

13. CASALINO E, SBLANO C AND LANDRISCINA C, 1997. Enzyme activity alteration by cadmium administration to rats: the possibility of iron involvement. Arch Biochem Biophy 346:

14. CHANEY R L, RYAN J A, LI Y M AND BROWN S L, 1999. Soil cadmium as a threat to human health. In: McLaughlin, M J, Singh, B R (Eds.), Developments in Plant and Soil Sciences, vol. 85. Kluwer Academic Publishers, Dordrecht, 219-256.

15. CHEN CY, ZHANG SL, LIU ZY, TIAN Y, SUN Q. Cadmium toxicity induces ER stress and apoptosis via impairing energy homeostasis in cardiomyocytes. Biosci Rep. 201510 (1) 52-9

16. CUYPERS A, PLUSQUIN M, REMANS T, JOZEFCZAK M, KEUNEN E, GIELEN H, OPDENAKKER K, NAIR A R, MUNTERS E, ARTOIS T J, NAWROT T, VANGRONSVELD J AND SMEETS K, 2010. Cadmium stress: an oxidative challenge. Biometals 23(5):927-40.

17. DIACOMANOLIS $\mathrm{V}$, NOLLER BN, NG JC. Bioavailability and pharmacokinetics of arsenic are influenced by the presence of cadmium. Chemosphere. 2014 Oct; 112:203-9. 
18. DOCAMPO A, GOMEZ V, LOPEZ R. Angiogenesis en la Hiperplasia de Células de Leydig inducida por cloruro de cadmio y estudio de Inmuno Histoquímico y Cuantitativo Depto de Morfología. Fac. Medicina UAM Madrid España; 2003

19. EL-SOKKARY GH, NAFADY AA, SHABASH EH. Melatonin administration ameliorates cadmiuminduced oxidative stress and morphological changes in the liver of rat. Ecotoxicol Environ Saf. 2010 Mar;73(3):456-63.

20. FIALA J, HRUBÁ D, CRHA I, RÉZL P AND TOTÛSEK J, 2001. Is environmental cadmium a serious hazard to Czech population? Int J Occup Med Environ Health 14:185-188.

21. FRIGERG L. Health hazards in the manufactures of alkaline accumulators with special reference to chronic cadmium poisoning. Act Med Scand 1980; 138: 1-124.

22. GARCÍA ORTIZ MESÍAS MOISÉS. Evaluación del riesgo por toxicidad crónica a la exposición de Cadmio en animales de experimentación. TESIS para optar al grado académico de Doctor en Farmacia y Bioquímica. UNMSM. 2008.

23. GOERING P L, WAALKES M P AND KLAASSEN C D, 1995.Toxicology of cadmium, in: Toxicology of Metals: Biochemical Aspects, Handbook of Experimental Pharmacology, Goyer RA, Cherian MG (eds), Springer, New York, 189-213.

24. GONZÁLEZ E. Toxicocinética y evaluación de riesgos para la salud producidos por exposición a cadmio. Medicina y Seguridad del Trabajo. 1988. XXXV: 3-17.

25. HAMADA T, TANIMOTO A AND SASAGURI Y, 1997. Apoptosis induced by cadmium. Apoptosis 2:359-367.

26. HIMENO S, YANAGIYA T, ENOMOTO S, KONDO Y AND IMURA N, 2002. Cellular cadmium uptake mediated by the transport system for manganese. Tohoku J Exp Med 196(1):43-

27. HUFF J, LUNN R M, WAALKES M P, TOMATIS L AND INFANTE P F, 2007. Cadmium-induced cancers in animals and in humans. Int J Occup Environ Health 13(2):202-12.

28. JIN T, LEFFLER P, NORDBERG GF. Cadmiummetallothionein nephrotoxicity in the rat; transient calciuria and proteinuria. Toxicology 1987; 45: 307-17.

29. KAWADA T, KOYAMA H, SUZUKI S. Cadmium, NAG activity, and B2M in the urine of cadmium pigment workers. Br J Ind Med 1989; 46: 52-5.

30. KIDO T, SHAIKH ZA, KITO H, HONDA R, NOGAWA K. Dose- response relationship between urinary cadmium and metallothionein in a Japanese population environmentally exposed to cadmium. Toxicology 1991; 65: 325-32.

31. KJELLSTROM T, NORDBERG GF. A kinetic model of Cd metabolism in the human being. Environ Res 1978; 16: 248-69.

32. KOLLER L D, 1998. Cadmium. In: Immunotoxicology of Environmental and Occupational

33. KORIEM KM, FATHI GE, SALEM HA, AKRAM NH, GAMIL SA. Protective role of pectin against cadmiuminduced testicular toxicity and oxidative stress in rats. Toxicol Mech Methods. 2013 May;23(4):263-72.

34. KORIEM KM, FATHI GE, SALEM HA, AKRAM NH,
GAMIL SA. Protective role of pectin against cadmiuminduced testicular toxicity and oxidative stress in rats. Toxicol Mech Methods. 2013 May;23(4):263-72.

35. LAFUENTE A, CANO P AND ESQUIFINO A, 2003. Are cadmium effects on plasma gonadotropins, prolactin, $\mathrm{ACTH}, \mathrm{GH}$ and $\mathrm{TSH}$ levels, dose-dependent? Biometals

36. LARA P, WATANABE L, PICOLI F. Efectos del Cadmio en la Mucosa Yugal de la rata durante la lactancia estudio morfológico e Histométrico. Int J. Morphol 2003,21(3):191-198

37. LASFER $\mathrm{M}$, VADROT $\mathrm{N}$, AOUDJEHANE L, CONTI F, BRINGUIER A F, FELDMANN G AND REYL- DESMARS F, 2008. Cadmium induces mitochondria-dependent apoptosis of normal human hepatocytes. Cell Biol Toxicol 24:55-62.

38. LEE YK, PARK EY, KIM S, SON JY, KIM TH, KANG WG, JEONG TC, KIM KB, KWACK SJ, LEE J, KIM S, LEE BM, KIM HS. Evaluation of cadmium-induced nephrotoxicity using urinary metabolomic profiles in Sprague-dawley male rats. J Toxicol Environ Health A. 2014;77(22-24):1384-98

39. LIU Y, WANG K, XU P AND WANG Z, 2012. Physiological responses and tolerance threshold to cadmium contamination in Eremochloa ophiuroides. Int J Phytoremediation 14(5):467-80.

40. LOPEZ E, ARCE C, OSET-GASQUE M J, CAÑADAS S AND GONZÁLEZ M P, 2006. Cadmium induces reactive oxygen species generation and lipid peroxidation in cortical neurons in culture. Free Radic Biol Med 40:940-951.

41. MADEJCZYK MS, BAER CE, DENNIS WE, MINARCHICK VC, LEONARD SS, JACKSON DA, STALLINGS JD, LEWIS JA. Temporal changes in rat liver gene expression after acute cadmium and chromium exposure. PLoS One. 2015 May 19;10(5).

42. MIURA NOBUHIKO, 2009. Individual susceptibility to cadmium toxicity and metallothionein gene polymorphisms: with references to current status of occupational cadmium exposure. Ind Health 47(5):487-94.

43. NASIADEK M, SKRZYPIŃSKA-GAWRYSIAK M, DARAGÓ A, ZWIERZYŃSKA E, KILANOWICZ A. Involvement of oxidative stress in the mechanism of cadmium-induced toxicity on rat uterus. EnvironToxicol Pharmacol. 2014 Jul 19;38(2):364-373.

44. OBIANIME AW, ROBERTS II. Antioxidants, cadmium-induced toxicity, serum biochemical and the histological abnormalities of the kidney and testes of the male Wistar rats. Niger J Physiol Sci. 2009 Dec;24(2):177-85.

45. OGNJANOVIĆ B I, MARKOVIĆ S D, PAVLOVIĆ S Z, ZIKIĆ R V, STAJN A S AND SAICIĆ Z S, 2008. Effect of chronic cadmium exposure on antioxidant defense system in some tissues of rats: protective effect of selenium. Physiol Res 57: 403-411.

46. PAKSY K, RAJCZY K, FORGÁCS Z, LÁZÁR P, BERNARD A, GÁTI I AND KAÁLI G S, 1997. Effect of cadmium on morphology and steroidogenesis of cultured human ovarian granulosa cells. J Appl Toxicol 17:321-327.

47. PETROCHELLI BANZATO T, GODINHO AF, 
DA SILVA ZACARIN EC, PEROBELLI JE, DAL BIANCO FERNANDEZ C, FAVARETO AP, DE GRAVA KEMPINAS W. Sperm quality in adult male rats exposed to cadmium in utero and lactation. $\mathrm{J}$ Toxicol Environ Health A. 2012;75(16-17):1047-58.

48. PIASEK M, BLANUSA M, KOSTIAL K AND LASKEY J W, 2001. Placental cadmium and progesterone concentrations in cigarette smokers. Reprod Toxicol 15:673-681.

49. PILLAI P, PATEL R, PANDYA C, GUPTA S. Sex-specific effects of gestational and lactational coexposure to lead and cadmium on hepatic phase I and phase II xenobiotic/steroid-metabolizing enzymes and antioxidant status. J Biochem Mol Toxicol. 2009 Nov;23(6):419-31.

50. POLIANDRI A, CABILLA P, VELARDEZ M, BODO C AND DUVILANSKI, 2003. Cadmium induces apoptosis in anterior pituitary cells that can be reversed by treatment with antioxidants. Toxicol Appl Pharmacol 190: 17-24.

51. RUBIN E. FARFER J. (2009) Essential Pathology, 3rd Ed. Lippincott Williams \& Wilkins, Philadelphia, PA.

52. SANCHO P, FERNÁNDEZ $\mathrm{C}$, YUSTE $\mathrm{V}$, AMRÁN D, RAMOS A M, DE BLAS E, SUSIN S A AND ALLER P, 2006. Regulation of apoptosis/ necrosis execution in cadmium-treated human promonocytic cells under different forms of oxidative stress. Apoptosis 11:673-686.

53. SHUKLA D, KESARI R, MISHRA S, DWIVEDI S, TRIPATHI R D, NATH P AND TRIVEDI P K, 2012. Expression of phytochelatin synthase from aquatic macrophyte Ceratophyllum demersum L. enhances cadmium and arsenic accumulation in tobacco. Plant Cell Rep [En prensa].

54. SIEWIT C L, GENGLER B, VEGAS E, PUCKETT R AND LOUIE M C, 2010. Cadmium promotes breast cancer cell proliferation by potentiating the interaction between ER alpha and c- Jun. Mol Endocrinol 24: 981992.

55. SUGITA M AND TSUCHIYA K, 1995. Estimation of variation among individuals of biological half-time of cadmium calculated from accumulation data. Environ Res 68(1):31-7.

56. WALLIN $\mathrm{M}$, SALLSTEN G, LUNDH $\mathrm{T}$, BARREGARD L. Low-level cadmium exposure and effects on kidney function. Occup Environ Med. 2014 Dec;71(12):848-54.

57. YANG H, SHU Y. Cadmium transporters in the kidney and cadmium-induced nephrotoxicity. Int J Mol Sci. 2015 Jan 9;16(1):1484-94.

58. YARI A, ASADI MH, BAHADORAN H, DASHTNAVARD H, IMANI H, NAGHII MR. Cadmium Toxicity in Spermatogenesis and Protective Effects of L: -Carnitine in Adult Male Rats. Biol Trace Elem Res. 2009 Dec 10.

59. ZADOROZHNAJA T D, LITTLE R E, MILLER R $\mathrm{K}$, MENDEL N A, TAYLOR R J, PRESLEY B J AND GLADEN B C, 2000. Concentrations of arsenic, cadmium, copper, lead, mercury, and zinc in human placentas from two cities in Ukraine. J Toxicol Environ Health 61:255-263.

60. ZHANG YM, LIU XZ, LU H, MEI L, LIU ZP. Lipid peroxidation and ultrastructural modifications in brain after perinatal exposure to lead and/or cadmium in rat pups. Biomed Environ Sci. 2009 Oct;22(5):423-9.

61. ZHANG YM, LIU XZ, LU H, MEI L, LIU ZP. Lipid peroxidation and ultrastructural modifications in brain after perinatal exposure to lead and/or cadmium in rat pups. Biomed Environ Sci. 2009 Oct;22(5):423-9. 